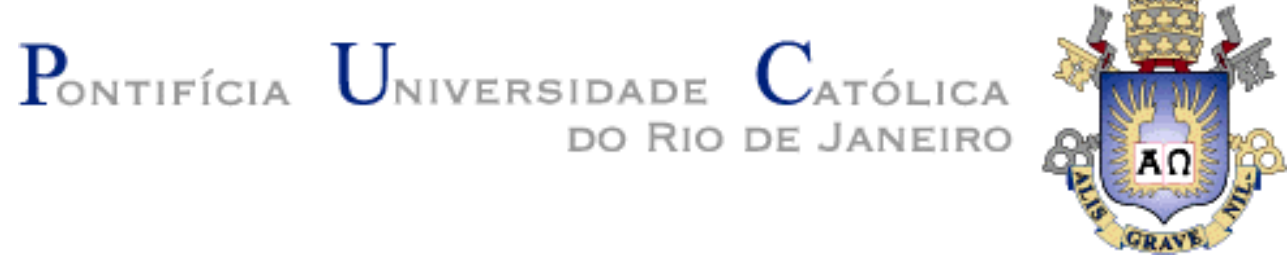

Rodrigo Corrêa Meirelles

\title{
A Digitalização da TV e os Contextos Culturais
}

Dissertação de Mestrado

Dissertação apresentada ao Programa de PósGraduação em Educação da PUC-Rio como requisito parcial para obtenção do título de Mestre em Educação.

Orientadora: Maria Apparecida Campos Mamede Neves

Rio de Janeiro

Junho de 2010 


\title{
Rodrigo Corrêa Meirelles
}

\section{A Digitalização da TV e os Contextos Culturais}

Dissertação apresentada como requisito parcial para obtenção do grau de Mestre pelo Programa de Pósgraduação em Educação do Departamento de Educação do Centro de Teologia e Ciências Humanas da PUC-Rio. Aprovada pela Comissão Examinadora abaixo assinada.

\author{
Prof $^{\text {a }}$. Maria Apparecida Campos Mamede Neves \\ Orientadora \\ PUC-Rio
}

Prof ${ }^{a}$. Stella Maria de Azevedo Pedrosa

Presidente

PUC-Rio

Prof. Ernani Ferraz

FACOM

Prof. Paulo Fernando Carneiro de Andrade Coordenador Setorial do Centro de Teologia e Ciências

Humanas

PUC-Rio

Rio de Janeiro, 18 de junho de 2010 
Todos os direitos reservados. É proibida a reprodução total ou parcial do trabalho sem autorização da universidade, do autor e da orientadora.

\section{Rodrigo Corrêa Meirelles}

Graduou-se em Engenharia Eletrônica e de Computação na UFRJ (Universidade Federal do Rio de Janeiro) em 2006. Na área de Engenharia desenvolveu pesquisas em áudio digital e atua como coordenador de engenharia de áudio na pós-produção da TV Globo. Sua experiência como professor universitário começou em 2002 na UNESA (Universidade Estácio de Sá), em cursos do instituto politécnico e graduação tecnológica.

Ficha Catalográfica

Meirelles, Rodrigo Corrêa

A digitalização da TV e os contextos culturais / Rodrigo Corrêa Meirelles ; orientadora: Maria Apparecida Campos Mamede Neves. - 2010.

129 f. ; $30 \mathrm{~cm}$

Dissertação (mestrado)-Pontifícia Universidade Católica do Rio de Janeiro, Departamento de Educação, 2010.

Inclui bibliografia

1. Educação - Teses. 2. TV digital e educação. 3. Interatividade. 4. Redes sociais. 5. Sociedade em rede. 6. TV interativa. 7. Portabilidade. 8. NTIC. I. Neves, Maria Apparecida Campos Mamede. II. Pontifícia Universidade Católica do Rio de Janeiro. Departamento de Educação. III. Título. 
Em memória de Edilson da Paz Corrêa e Gilberte Cardoso. 


\section{Agradecimentos}

Agradeço à minha mãe pelo carinho, referência e suporte de sempre, ao meu pai pela construção de valores que preservo como presente eterno; à minha família pelos momentos de felicidade e reflexão. À minha companheira Ana Maria agradeço pelo apoio incondicional, fundamental desde o início de toda essa jornada, da seleção à conclusão da dissertação; e aos meus amigos por entenderem e darem força em meus freqüêntes momentos de ausência.

À minha orientadora Maria Apparecida Mamede, ficam os agradecimentos e a admiração por acreditar sempre no tema e apostar no meu trabalho. Cada cobrança e frase de motivação foram fundamentais para o cumprimento das metas necessárias. Aos colegas da turma de mestrandos 2008, agradeço pelo suporte e construção coletiva de conhecimento durante as aulas; aos colegas de trabalho, pela ajuda mútua nos momentos difíceis. À CAPES, agradeço pelo investimento durante os poucos meses de bolsa de fomento, essenciais para a establilidade em uma fase financeiramente conturbada. Ao Programa de Pós-Graduação do Departamento de Educação da PUC-Rio, meus sinceros agradecimentos por acolher tão bem um engenheiro eletrônico de graduação que tem a certeza de que aprendeu muito e conseguiu retribuir com o melhor que poderia para o campo do conhecimento qual se orgulha em fazer parte. Abrir espaço para a troca de conhecimentos e à interdisciplinaridade na prática me fez admirar e respeitar ainda mais a equipe que faz deste Programa que é referência.

Agradeço e também dedico este trabalho aos profissionais que foram de fundamental importância para a construção e conclusão desta pesquisa: Mayrton Bahia, Paulo Henrique Castro, Fred Moussalem, Filipe Diniz, Tatiane Martins, Luiz Alexandre Rosado, Mauricio Castanheira, Luiz Carlos Esteves, Luiz Wagner Biscainho, Álvaro Veiga, Newton Canitto, Claudia Elias, Izaias Silva, Fátima Alves, Rosalia Duarte, Zaia Brandão, Zena Eisenberg, Ana Waleska, Isabel Lelis e outros que por ventura eu tenha cometido o erro de não ter mencionado, mas sabem o quanto sou grato pelo apoio. 


\section{Resumo}

Meirelles, Rodrigo C.; Mamede, Maria Apparecida C. Neves. A Digitalização da TV e os Contextos Culturais. Rio de Janeiro, 2010. 129p. Dissertação de Mestrado - Departamento de Educação, Pontifícia Universidade Católica do Rio de Janeiro.

Este trabalho tem como objetivo estudar a introdução da TV Digital terrestre no Brasil sob os aspectos de Educação e Cultura. Voltar o olhar para as instâncias culturais que mediam tal transição foi considerado fundamental, uma vez que os pensadores de televisão, seja no plano mercadológico, seja no campo acadêmico, enxergam no recém-implantado e já em operação Sistema Brasileiro de TV Digital terrestre (SBTVD-t), uma oportunidade para a inclusão digital, para a criação de novos conteúdos e para a convergência com outras mídias digitais. A pesquisa teve como fontes de consulta documentos oficiais, trabalhos publicados, entrevistas com especialistas e participação em conferências internacionais sobre o tema. As representações sociais, nos termos propostos por Moskovici e Jodelet, dadas à TVD por professores e estudantes foi também outro campo de investigação. A análise do material permitiu o surgimento de quatro eixos temáticos: alta-definição, multiprogramação, interatividade e portabilidade, sendo que neste trabalho é dada ênfase aos dois últimos. As conclusões mostram que existem muitas expectativas em relação à TV Digital, embora atualmente ainda não passe de sinônimo de alta definição de imagem e som. No sentido de mudanças significativas, a TV tem seu papel a ser repensado entre os meios de comunicação, principalmente quando relacionados à educação, permanecendo a incógnita de qual função social a TV Digital passaria a ter no contexto dos modos de recepção das novas mídias digitais como a interatividade da internet, redes sociais virtuais e vídeos sob demanda. Finalmente, a pesquisa aponta para necessidade de novas investigações que avancem no sentido da reverberação das vozes do campo da educação sobre esse que é um dos temas mais relevantes na discussão atual de mídia no Brasil.

\section{Palavras-chave}

TV Digital e educação; interatividade; redes sociais; sociedade em rede; TV interativa; portabilidade; NTIC; inteligência coletiva; transmídia; SBTVD; cultura da convergência; Ginga; educação e mídia; DTVi; transição digital. 


\section{Abstract}

Meirelles, Rodrigo C.; Mamede, Maria Apparecida C. Neves (Advisor). TV Digitization and the Cultural Contexts. Rio de Janeiro, 2010. 129p. MSc. Dissertation - Departamento de Educação, Pontifícia Universidade Católica do Rio de Janeiro.

This work aims to study the introduction of digital TV in Brazil under the aspects of education and culture. To examine the cultural instances that mediate this transition was considered crucial, since both in the marketplace and in academia, the newly established, operating Brazilian System of Digital Terrestrial TV (SBTVD-t) is seen as an opportunity for digital inclusion, as well as for creating new content and bring about synergies with other digital media. The research is based on official documents, published papers, interviews with experts, and participation in international conferences on the subject. The study of teachers's and students's "social representations" of digital TV constituted another subject of the present research. Four themes emerged through the analysis of the material: high definition, multiprogramming, portability and interactivity. Emphasis was given to the last two. The conclusions show that while expectations are extremely positive, digital TV currently is no more than a synonym of high-definition for picture and sound. Its role has to be rethought in the communication scenario, especially in relation to education. Moreover, its social function still needs to be better understood in the context of the reception of new digital media (e.g. internet interactivity of the Internet, virtual social networks, video on demand). Finally, the research points to the need for further research to move towards more connections with the field of education, which is central in discussions about the media in Brazil.

\section{Keywords}

Digital TV and education; interactivity; social networks; network society; interactive TV; portability; ICT; collective intelligence; transmedia; ISDB-Tb; convergence culture; Ginga; education and media; DTVi; digital transition. 


\section{Sumário}

1. Introdução 10

$\begin{array}{ll}1.1 \text { Metodologia } & 11\end{array}$

2. Uma TV pensada para a educação e a cultura 15

2.1. Mídias e TVs Digitais - esclarecimentos e inquietações 16

2.2. A TV Digital e a Educação - para além da EAD 21

3. A TV e as mídias do Século XXI 25

3.1. A metamorfose da televisão no Brasil. 30

3.2. Políticas públicas relacionadas 33

3.3. A convergência e a transmídia como novos conceitos da mídia no século XXI 35

3.4. A TV Digital brasileira e a cultura da convergência 38

4. A TV Interativa $\quad 41$

4.1. Inquietações interativas em televisão. $\quad 42$

4.2. O conceito de Interatividade. 44

4.2.1. O conceito de interatividade e as mídias digitais 45

4.3. A TV sempre foi interativa? 52

4.4. E a TV Digital Interativa? 56

5. A interatividade no SBTVD 61

5.1. O Cenário Atual $\quad 62$

5.2. Possibilidades do SBTVD 65

5.2.1. Interatividade Local. 66

5.2.2. Interatividade via "canal de retorno". $\quad 69$

5.3. Conteúdos interativos - perspectivas e dilemas 72

5.4. TV e internet: casamento ou concorrência?

6. Portabilidade, Convergência e Transmídia - a TV como parte de novos $\begin{array}{ll}\text { contextos } & 91\end{array}$

6.1. Portabilidade, mobilidade e a sociedade em rede 93

$\begin{array}{ll}\text { 6.2. Debates sobre a portabilidade. } & 97\end{array}$ 
6.3. Portabilidade, convergência e novas formas de narrativa: a emergência da transmídia

7. Conclusões

8. Referências Bibliográficas 